TAMKANG JOURNAL OF MATHEMATICS

Volume 21, Number 2, Summer 1990

\title{
ON INTEGRAL INEQUALITIES RELATED TO OPIAI'S INEQUALITY
}

\author{
TIAN-SHOW HWANG AND GOU-SHENG YANG
}

\section{Introduction.}

In 1960, Z. Opial [4] proved the following integral inequality:

Theorem $\mathbb{A}$. Let $u$ be of class $c^{1}$ on $[0, b]$, and satisfy $u(0)=u(b)=0, u>0$ on $(0, b)$. Then

$$
\int_{0}^{b}\left|u(x) u^{\prime}(x)\right| d x \leq \frac{b}{4} \int_{0}^{b}\left|u^{\prime}(x)\right|^{2} d x
$$

where the constant $b / 4$ is the best possible.

We note that if we replaced $u(0)=u(b)=0$ by $u(0)=0$ (or $u(b)=0$ ), then (1) becomes

$$
\int_{0}^{b}\left|u(x) u^{\prime}(x)\right| d x \leq \frac{b}{2} \int_{0}^{b}\left|u^{\prime}(x)\right|^{2} d x
$$

C. Olech [5] showed that (1) is valid for any function $u$ which is absolutely continuous on $[0, b]$, and satisfies the boundary conditions $u(0)=u(b)=0$, and Olech's proof of (1) was simplier than that of Opial.

In 1967, E.K.Godunova, and V.I.Levin [1] generalized $\left(1^{\prime}\right)$ and $(1)$ in the following forms:

Theorem B. Let $u$ be absolutely continuous on $[a, b]$ with $u(a)=0$. If $f$ is convex increasing on $[0, \infty)$ and $f(0)=0$. Then

$$
\int_{a}^{b} f^{\prime}(|u(x)|)\left|u^{\prime}(x)\right| d x \leq f\left(\int_{a}^{b}\left|u^{\prime}(x)\right| d x\right)
$$

Theorem C. Let $u$ be absolutely continuous on $[a, b]$ with $u(a)=u(b)=0$. Let $p$ be positive on $[a, b]$ and $\int_{a}^{b} p(x) d x=1$, and let $f, h$ be convex increasing on $(0, \infty)$, and $f(0)=0$. Then

$$
\int_{a}^{b} f^{\prime}(|u(x)|)\left|u^{\prime}(x)\right| d x \leq 2 f\left(h^{-1}\left(\int_{a}^{b} p(x) h\left(\frac{\left|u^{\prime}(x)\right|}{2 p(x)}\right) d x\right)\right)
$$

In 1966, G.S.Yang [6] generalized $\left(1^{\prime}\right)$ and (1) in the following forms:

Received Oct. 14, 1986; revised March, 23, 1987. 
Theorem $\mathbb{D}$. If $u$ is absolutely continuous on $[a, b]$ with $u(a)=0$, then

$$
\int_{a}^{b}|u(x)|^{m}\left|u^{\prime}(x)\right|^{n} d x \leq \frac{n}{m+n}(b-a)^{m} \int_{a}^{b}\left|u^{\prime}(x)\right|^{m+n} d x
$$

where $m \geq 0, n \geq 1$.

Theorem $\mathbb{E}$. If $u$ is absolutely continuous on $[a, b]$ with $u(a)=u(b)=0$, then

$$
\int_{a}^{b}|u(x)|^{m}\left|u^{\prime}(x)\right|^{n} d x \leq \frac{n}{m+n}\left(\frac{b-a}{2}\right)^{m} \int_{a}^{b}\left|u^{\prime}(x)\right|^{m+n} d x
$$

where $m \geq 0, n \geq 1$.

Theorem $\mathrm{F}$. Let $p$ be positive on $[a, b]$ with $\int_{a}^{b} \frac{1}{p(x)} d x<\infty$, and let $q$ be positive, bounded and nonincreasing on $[a, b]$. If $u$ is absolutely continuous on $[a, b]$ with $u(a)=0$, then

$$
2 \int_{a}^{b} q(x)|u(x)|\left|u^{\prime}(x)\right| d x \leq\left(\int_{a}^{b} \frac{1}{p(x)} d x\right)\left(\int_{a}^{b} p(x) q(x)\left|u^{\prime}(x)\right|^{2} d x\right)
$$

The aim of this paper is to establish some new integral inequalities which generalize $(2),(3),(4),(5)$, and (6).

\section{Main results}

Throughout, we assume that $n$ is a real number such that $n \geq 1$, and $k=(b-a)^{n-1}$.

Theorem 1. Let $u_{1}, u_{2}$ be absolutely continuous on $[a, b]$ with $u_{1}(a)=u_{2}(a)=0$. Let $f_{1}, f_{2}$ be nonnegative, continuous on $[0, \infty)$ with $f_{1}(0)=0$ such that $f_{1}^{\prime}, f_{2}^{\prime}$ exist, nonnegative, continuous, and nondecreasing on $[0, \infty)$. Then

$$
\begin{gathered}
\int_{a}^{b}\left[f_{1}\left(\left|u_{1}(x)\right|^{n}\right) f_{2}^{\prime}\left(\left|u_{2}^{\prime}(x)\right|^{n}\right)\left|u_{2}^{\prime}(x)\right|^{n}+f_{2}\left(\left|u_{2}(x)\right|^{n}\right) f_{1}^{\prime}\left(\left|u_{1}(x)\right|^{n}\right)\left|u_{1}^{\prime}(x)\right|^{n}\right] d x \\
\quad \leq \frac{1}{k} f_{1}\left(k \int_{a}^{b}\left|u_{1}^{\prime}(x)\right|^{n} d x\right) f_{2}\left(k \int_{a}^{b}\left|u_{2}^{\prime}(x)\right|^{n} d x\right)
\end{gathered}
$$

Proof. For $x \in[a, b]$, and $i=1,2$, define

$$
y_{i}(x)=\int_{a}^{x}\left|u_{i}^{\prime}(x)\right|^{n} d x \text {. }
$$

Then $y_{i}^{\prime}(x)=\left|u_{i}^{\prime}(x)\right|^{n}$, and using Holder inequality, we have

$$
\left|u_{i}(x)\right|^{n} \leq\left(\int_{a}^{x}\left|u_{i}^{\prime}(x)\right| d x\right)^{n} \leq\{b-a\}^{(n-1)}\left(\int_{a}^{x}\left|u_{i}^{\prime}(x)\right|^{n} d x\right) \leq k y_{i}(x) .
$$


Since $f_{i}, f_{i}^{\prime}$ are nondecreasing, nonnegative, and continuous on $[0, \infty)$, we have

$$
\begin{aligned}
& \int_{a}^{b}\left[f_{1}\left(\left|u_{1}(x)\right|^{n}\right) f_{2}^{\prime}\left(\left|u_{2}(x)\right|^{n}\right)\left|u_{2}^{\prime}(x)\right|^{n}+f_{2}\left(\left|u_{2}(x)\right|^{n}\right) f_{1}^{\prime}\left(\left|u_{1}(x)\right|^{n}\right)\left|u_{1}^{\prime}(x)\right|^{n}\right] d x \\
& \leq \int_{a}^{b}\left[f_{1}\left(k y_{1}\right) f_{2}^{\prime}\left(k y_{2}\right) y_{2}^{b}+f_{2}\left(k y_{2}\right) f_{1}^{\prime}\left(k y_{1}\right) y_{1}^{\prime}\right] d x \\
& =\frac{1}{k} \int_{a}^{b} \frac{d}{d x}\left[f_{1}\left(k y_{1}\right) f_{2}\left(k y_{2}\right)\right] d x \\
& =\frac{1}{k} f_{1}\left(k \int_{a}^{b}\left|u_{1}^{\prime}(x)\right|^{n} d x\right) f_{2}\left(k \int_{a}^{b}\left|u_{2}^{b}(x)\right|^{n} d x\right)
\end{aligned}
$$

This completes the proof of Theorem 1.

Remark.

1. Let $n=1$, and $f_{1}(x)=f(x), f_{2}(x)=1, u_{1}(x)=u_{2}(x)=u(x)$ in Theorem 1 . Then, it follows from (7) that

$$
\int_{a}^{b} f^{\prime}(|u(x)|)\left|u^{\prime}(x)\right| d x \leq f\left(\int_{a}^{b}\left|u^{\prime}(x)\right| d x\right)
$$

which is the inequality (2). [ See also [2], p159, Theorem 13 ]

2. Let $f_{1}(x)=x^{(m+n) / n}$, where $m \geq 0$, and let $f_{2}(x)=1, u_{1}(x)=u_{2}(x)=u(x)$ in Theorem 1. Then it follows from (7), and Holder inequality that

$$
\begin{aligned}
\int_{a}^{b}|u(x)|^{m}\left|u^{\prime}(x)\right|^{n} d x & \leq \frac{n}{m+n} \frac{1}{k}\left\{k \int_{a}^{b}\left|u^{\prime}(x)\right|^{n} d x\right\}^{(m+n) / n} \\
& \leq \frac{n}{m+n} k^{\frac{m}{m}}\left(\int_{a}^{b} d x\right)^{\frac{m}{m}}\left(\int_{a}^{b}\left|u^{\prime}(x)\right|^{m+n} d x\right) \\
& =\frac{n}{m+n}(b-a)^{m} \int_{a}^{b}\left|u^{\prime}(x)\right|^{m+n} d x,
\end{aligned}
$$

which is the inequality (4). [See [6], Lemma 7]

3. If $q$ is a positive, bounded, and nonincreasing function on $[a, b]$, and $p$ is a positive function with $\int_{a}^{b} \frac{1}{p(x)} d x<\infty$, let $f_{1}(x)=x^{2}, f_{2}(x)=1, n=1$, and $u_{1}(x)=u_{2}(x)=$ $\int_{a}^{2} \sqrt{q(t)}\left|u^{\prime}(t)\right| d t$ in Theorem 1. We have

$$
2 \int_{a}^{b}\left(\int_{a}^{2} \sqrt{q(t)}\left|u^{\prime}(t)\right| d t\right) \sqrt{q(x)}\left|u^{\prime}(x)\right| d x \leq\left(\int_{a}^{b} \sqrt{q(x)}\left|u^{\prime}(x)\right| d x\right)^{2}
$$

Since $q$ is nonincreasing, we have

$$
\int_{a}^{x} \sqrt{q(t)}\left|u^{\prime}(t)\right| d t \geq \sqrt{q(x)} \int_{a}^{x}\left|u^{\prime}(t)\right| d t \geq\left|\sqrt{q(x)} \int_{a}^{x} u^{\prime}(t) d t\right|=\sqrt{q(x)}|u(x)|
$$


Thus

$$
\begin{aligned}
2 \int_{a}^{b} q(x)|u(x)|\left|u^{\prime}(x)\right| d x & \leq 2 \int_{a}^{b}\left(\int_{a}^{x} \sqrt{q(t)}\left|u^{\prime}(t)\right| d t\right) \sqrt{q(x)}\left|u^{\prime}(x)\right| d x \\
& \leq\left(\int_{a}^{b} \sqrt{q(x)}\left|u^{\prime}(x)\right| d x\right)^{2} \\
& =\left(\int_{a}^{b} \frac{1}{\sqrt{p(x)}} \sqrt{p(x)} \sqrt{q(x)}\left|u^{\prime}(x)\right| d x\right)^{2} \\
& \leq\left(\int_{a}^{b} \frac{1}{p(x)} d x\right)\left(\int_{a}^{b} p(x) q(x)\left|u^{\prime}(x)\right|^{2} d x\right)
\end{aligned}
$$

which is the inequality (6). [See [6], Theorem 3 ]

Theorem 2. For $i=1,2$, let $u_{i}, f_{i}, f_{i}^{\prime}$ be as in Theorem 1. Let $p_{i}$ be positive on $[a, b]$, and $\int_{a}^{b} p_{i}(x) d x=1$. If $h$ is a positive, convex, and increasing function on $[0, \infty)$, then

$$
\begin{aligned}
& \int_{a}^{b}\left[f_{1}\left(\left|u_{1}(x)\right|^{n}\right) f_{2}^{\prime}\left(\left|u_{2}(x)\right|^{n}\right)\left|u_{2}^{\prime}(x)\right|^{n}+f_{2}\left(\left|u_{2}(x)\right|^{n}\right) f_{1}^{\prime}\left(\left|u_{1}(x)\right|^{n}\right)\left|u_{1}^{\prime}(x)\right|^{n}\right] d x \\
& \leq \frac{1}{k} f_{1}\left(2 k h^{-1}\left(\int_{a}^{b} p_{1}(x) h\left(\frac{\left|u_{1}^{\prime}(x)\right|^{n}}{2 p_{1}(x)}\right) d x\right)\right) f_{2}\left(2 k h^{-1}\left(\int_{a}^{b} p_{2}(x) h\left(\frac{\left|u_{2}^{\prime}(x)\right|^{n}}{2 p_{2}(x)}\right) d x\right)\right)
\end{aligned}
$$

Proof. For $i=1,2$, it follows from Jensen's inequality, that

$$
h\left(\frac{1}{2} \int_{a}^{b}\left|u_{i}^{\prime}(x)\right|^{n} d x\right) \leq \int_{a}^{b} p_{i}(x) h\left(\frac{\left|u_{i}^{\prime}(x)\right|^{n}}{2 p_{i}(x)}\right) d x .
$$

Since $h$ is increasing, so that

$$
\int_{a}^{b}\left|u_{i}^{\prime}(x)\right|^{n} d x \leq 2 h^{-1}\left(\int_{a}^{b} p_{i}(x) h\left(\frac{\left|u_{i}^{\prime}(x)\right|^{n}}{2 p_{i}(x)}\right) d x\right),
$$

which together with Theorem 1 imply that

$$
\begin{aligned}
& \int_{a}^{b}\left[f_{1}\left(\left|u_{1}(x)\right|^{n}\right) f_{2}^{\prime}\left(\left|u_{2}(x)\right|^{n}\right)\left|u_{2}^{\prime}(x)\right|^{n}+f_{2}\left(\left|u_{2}(x)\right|^{n}\right) f_{1}^{\prime}\left(\left|u_{1}(x)\right|^{n}\right)\left|u_{1}^{\prime}(x)\right|^{n}\right] d x \\
& \leq \frac{1}{k} f_{1}\left(2 k h^{-1}\left(\int_{a}^{b} p_{1}(x) h\left(\frac{\left|u_{1}^{\prime}(x)\right|^{n}}{2 p_{1}(x)}\right) d x\right)\right) f_{2}\left(2 k h^{-1}\left(\int_{a}^{b} p_{2}(x) h\left(\frac{\left|u_{2}^{\prime}(x)\right|^{n}}{2 p_{2}(x)}\right) d x\right)\right)
\end{aligned}
$$

This is the desired inequality.

Theorem 3. For $i=1,2$, let $u_{i}$ be absolutely continuous functions on $[a, b]$ with $u_{i}(a)=u_{i}(b)=0$. Let $f_{i}$ be nonnegative, continuous, nondecreasing functions on $[0, \infty)$ 
with $f_{1}(0)=0$ and that $f_{i}^{\prime}$ exist, nonnegative, continuous, and nondecreasing on $[0, \infty)$. Then

$$
\begin{aligned}
& \int_{a}^{b}\left[f_{1}\left(\left|u_{1}(x)\right|^{n}\right) f_{2}^{\prime}\left(\left|u_{2}(x)\right|^{n}\right)\left|u_{2}^{\prime}(x)\right|^{n}+f_{2}\left(\left|u_{2}(x)\right|^{n}\right) f_{1}^{\prime}\left(\left|u_{1}(x)\right|^{n}\right)\left|u_{1}^{\prime}(x)\right|^{n}\right] d x \\
& \leq \frac{2^{n-1}}{k} f_{2}\left(2^{1-n} k \int_{a}^{b}\left|u_{2}^{\prime}(x)\right|^{n} d x\right)\left[f_{1}\left(2^{1-n} k \int_{a}^{(a+b) / 2}\left|u_{1}^{\prime}(x)\right|^{n} d x\right)\right. \\
& \left.\quad+f_{1}\left(2^{1-n} k \int_{(a+b) / 2}^{b}\left|u_{1}^{\prime}(x)\right|^{n} d x\right)\right]
\end{aligned}
$$

and

$$
\begin{aligned}
& \int_{a}^{b}\left[f_{1}\left(\left|u_{1}(x)\right|^{n}\right) f_{2}^{\prime}\left(\left|u_{2}(x)\right|^{n}\right)\left|u_{2}^{\prime}(x)\right|^{n}+f_{2}\left(\left|u_{2}(x)\right|^{n}\right) f_{1}^{\prime}\left(\left|u_{1}(x)\right|^{n}\right)\left|u_{1}^{\prime}(x)\right|^{n}\right] d x \\
& \leq \frac{2}{k} f_{1}\left(\frac{k}{2} \int_{a}^{b}\left|u_{1}^{\prime}(x)\right|^{n} d x\right) f_{2}\left(k \int_{a}^{b}\left|u_{2}^{\prime}(x)\right|^{n} d x\right)
\end{aligned}
$$

Proof. For $i=1,2$, we note first that, by defining $y_{i}(x)=\int_{x}^{b}\left|u_{i}^{\prime}(x)\right|^{n} d x$, and using a similar argument as in the proof of Theorem 1 . We see that the inequality (1) still holds if we replaced $u_{1}(a)=u_{2}(a)=0$ by $u_{1}(b)=u_{2}(b)=0$. Now for any $c \in(a, b)$, we have

$$
\begin{aligned}
& \int_{a}^{c}\left[f_{1}\left(\left|u_{1}(x)\right|^{n}\right) f_{2}^{\prime}\left(\left|u_{2}(x)\right|^{n}\right)\left|u_{2}^{\prime}(x)\right|^{n}+f_{2}\left(\left|u_{2}(x)\right|^{n}\right) f_{1}^{\prime}\left(\left|u_{1}(x)\right|^{n}\right)\left|u_{1}^{\prime}(x)\right|^{n}\right] d x \\
& \leq \frac{1}{k_{1}} f_{1}\left(k_{1} \int_{a}^{c}\left|u_{1}^{\prime}(x)\right|^{n} d x\right) f_{2}\left(k_{1} \int_{a}^{c}\left|u_{2}^{\prime}(x)\right|^{n} d x\right)
\end{aligned}
$$

where $k_{1}=(c-a)^{n-1}$, and

$$
\begin{aligned}
& \int_{c}^{b}\left[f_{1}\left(\left|u_{1}(x)\right|^{n}\right) f_{2}^{\prime}\left(\left|u_{2}(x)\right|^{n}\right)\left|u_{2}^{\prime}(x)\right|^{n}+f_{2}\left(\left|u_{2}(x)\right|^{n}\right) f_{1}^{\prime}\left(\left|u_{1}(x)\right|^{n}\right)\left|u_{1}^{\prime}(x)\right|^{n}\right] d x \\
& \leq \frac{1}{k_{2}} f_{1}\left(k_{2} \int_{c}^{b}\left|u_{1}^{\prime}(x)\right|^{n} d x\right) f_{2}\left(k_{2} \int_{c}^{b}\left|u_{2}^{\prime}(x)\right|^{n} d x\right)
\end{aligned}
$$

where $k_{2}=(b-c)^{n-1}$.

Hence

$$
\begin{aligned}
& \int_{a}^{b}\left[f_{1}\left(\left|u_{1}(x)\right|^{n}\right) f_{2}^{\prime}\left(\left|u_{2}(x)\right|^{n}\right)\left|u_{2}^{\prime}(x)\right|^{n}+f_{2}\left(\left|u_{2}(x)\right|^{n}\right) f_{1}^{\prime}\left(\left|u_{1}(x)\right|^{n}\right)\left|u_{1}^{\prime}(x)\right|^{n}\right] d x \\
& \leq \frac{1}{k_{1}} f_{1}\left(k_{1} \int_{a}^{c}\left|u_{1}^{\prime}(x)\right|^{n} d x\right) f_{2}\left(k_{1} \int_{a}^{c}\left|u_{2}^{\prime}(x)\right|^{n} d x\right) \\
& \quad+\frac{1}{k_{2}} f_{1}\left(k_{2} \int_{c}^{b}\left|u_{1}^{\prime}(x)\right|^{n} d x\right) f_{2}\left(k_{2} \int_{c}^{b}\left|u_{2}^{\prime}(x)\right|^{n} d x\right)
\end{aligned}
$$


By taking $c=\frac{(a+b)}{2}$ in $(10)$, we have $k_{1}=k_{2}=2^{1-n} k$, and since $f_{2}$ is nondecreasing, so that

$$
\begin{aligned}
& \int_{a}^{b}\left[f_{1}\left(\left|u_{1}(x)\right|^{n}\right) f_{2}^{\prime}\left(\left|u_{2}(x)\right|^{n}\right)\left|u_{2}^{\prime}(x)\right|^{n}+f_{2}\left(\left|u_{2}(x)\right|^{n}\right) f_{1}^{\prime}\left(\left|u_{1}(x)\right|^{n}\right)\left|u_{1}^{\prime}(x)\right|^{n}\right] d x \\
& \leq \frac{2^{n-1}}{k} f_{2}\left(2^{1-n} k \int_{a}^{b}\left|u_{2}^{\prime}(x)\right|^{n} d x\right)\left[f_{1}\left(2^{1-n} k \int_{a}^{(\infty+b) / 2}\left|u_{1}^{\prime}(x)\right|^{n} d x\right)\right. \\
& \left.\quad+f_{1}\left(2^{1-n} k \int_{(a+b) / 2}^{b}\left|u_{1}^{\prime}(x)\right|^{n} d x\right)\right]
\end{aligned}
$$

This is the inequality (8).

If we choose $c$ in (4) so that

$$
\int_{a}^{c}\left|u_{1}^{\prime}(x)\right|^{n} d x=\int_{c}^{b}\left|u_{1}^{\prime}(x)\right|^{n} d x=\frac{1}{2} \int_{a}^{b}\left|u_{1}^{0}(x)\right|^{n} d x
$$

then,

$$
\begin{aligned}
& \int_{a}^{b}\left[f_{1}\left(\left|u_{1}(x)\right|^{n}\right) f_{2}^{\prime}\left(\left|u_{2}(x)\right|^{n}\right)\left|u_{2}^{\prime}(x)\right|^{n}+f_{2}\left(\left|u_{2}(x)\right|^{n}\right) f_{1}^{\prime}\left(\left|u_{1}(x)\right|^{n}\right)\left|u_{1}^{\prime}(x)\right|^{n}\right] d x \\
& \leq \frac{1}{k_{1}} f_{1}\left(\frac{k_{1}}{2} \int_{a}^{b}\left|u_{1}^{\prime}(x)\right|^{n} d x\right) f_{2}\left(k_{1} \int_{a}^{c}\left|u_{2}^{\prime}(x)\right|^{n} d x\right) \\
& \quad+\frac{1}{k_{2}} f_{1}\left(\frac{k_{2}}{2} \int_{a}^{b}\left|u_{1}^{\prime}(x)\right|^{n} d x\right) f_{2}\left(k_{2} \int_{c}^{b}\left|u_{2}^{\prime}(x)\right|^{n} d x\right) \\
& \leq f_{2}\left(k \int_{a}^{b}\left|u_{2}^{\prime}(x)\right|^{n} d x\right)\left[\frac{1}{k_{1}} f_{1}\left(\frac{k_{1}}{2} \int_{a}^{b}\left|u_{1}^{\prime}(x)\right|^{n} d x\right)\right. \\
& \left.\quad+\frac{1}{k_{2}} f_{1}\left(\frac{k_{2}}{2} \int_{a}^{b}\left|u_{1}^{\prime}(x)\right|^{n} d x\right)\right] \\
& =f_{2}\left(k \int_{a}^{b}\left|u_{2}^{\prime}(x)\right|^{n} d x\right)\left[\frac{1}{k_{1}} f_{1}\left(\frac{k_{1}}{k} \frac{k}{2} \int_{a}^{b}\left|u_{1}^{\prime}(x)\right|^{n} d x\right)\right. \\
& \left.\quad+\frac{1}{k_{2}} f_{1}\left(\frac{k_{2}}{k} \frac{k}{2} \int_{a}^{b}\left|u_{1}^{\prime}(x)\right|^{n} d x\right)\right] \\
& \leq \frac{2}{k} f_{1}\left(\frac{k}{2} \int_{a}^{b}\left|u_{1}^{\prime}(x)\right|^{n} d x\right) f_{2}\left(k \int_{a}^{b}\left|u_{2}^{\prime}(x)\right|^{n} d x\right)
\end{aligned}
$$

This completes the proof of Theorem 3.

Remark. For $m \geq 0$, let $f_{1}(x)=x^{(m+n) / n}, f_{2}(x)=1, u_{1}(x)=u_{2}(x)=u(x)$ in Theorem 3 , it follows from (8), and Holder inequality that

$$
\int_{a}^{b}|u(x)|^{m}\left|u^{\prime}(x)\right|^{n} d x \leq \frac{n}{m+n}\left(\frac{b-a}{2}\right)^{m} \int_{a}^{b}\left|u^{\prime}(x)\right|^{m+n} d x,
$$

which is the inequality (5). [ See [6], Theorem 6] 
Theorem 4. For $i=1,2$, let $u_{i}, f_{i}, f_{i}^{\prime}$ be as in Theorem 3. Let $p_{i}$ be positive on $[a, b]$, and $\int_{a}^{b} p_{i}(x) d x=1$. If $h$ is a positive, convex, and increasing function on $[0, \infty)$, then

$$
\begin{aligned}
& \int_{a}^{b}\left[f_{1}\left(\left|u_{1}(x)\right|^{n}\right) f_{2}^{\prime}\left(\left|u_{2}(x)\right|^{n}\right)\left|u_{2}^{\prime}(x)\right|^{n}+f_{2}\left(\left|u_{2}(x)\right|^{n}\right) f_{1}^{\prime}\left(\left|u_{1}(x)\right|^{n}\right)\left|u_{1}^{\prime}(x)\right|^{n}\right] d x \\
& \leq \frac{2}{k} f_{1}\left(k h^{-1}\left(\int_{a}^{b} p_{1}(x) h\left(\frac{\left|u_{1}^{\prime}(x)\right|^{n}}{2 p_{1}(x)}\right) d x\right)\right) f_{2}\left(2 k h^{-1}\left(\int_{a}^{b} p_{2}(x) h\left(\frac{\left|u_{2}^{\prime}(x)\right|^{n}}{2 p_{2}(x)}\right) d x\right)\right)
\end{aligned}
$$

Proof. For $i=1,2$, it follows from Jensen's inequality, that

$$
h\left(\frac{1}{2} \int_{a}^{b}\left|u_{i}^{\prime}(x)\right|^{n} \cdot d x\right) \leq \int_{a}^{b} p_{i}(x) h\left(\frac{\left|u_{i}^{\prime}(x)\right|^{n}}{2 p_{i}(x)}\right) d x .
$$

Since $h$ is increasing, we have

$$
\int_{a}^{b}\left|u_{i}^{\prime}(x)\right|^{n} d x \leq 2 h^{-1}\left(\int_{a}^{b} p_{i}(x) h\left(\frac{\left|u_{i}^{\prime}(x)\right|^{n}}{2 p_{i}(x)}\right) d x\right) .
$$

The desired inequality (11) is then follows from (9), and (12).

Remark. For $i=1,2$, let $p_{i}(x)=p(x), u_{i}(x)=u(x)$, and let $n=1, f_{1}(x)=$ $f(x), f_{2}(x)=1$ in Theorem 4 . Then it follows from the inequality (11) that

$$
\int_{a}^{b} f^{\prime}(|u(x)|)\left|u^{\prime}(x)\right| d x \leq 2 f\left(h^{-1}\left(\int_{a}^{b} p(x) h\left(\frac{\left|u^{\prime}(x)\right|}{2 p(x)}\right) d x\right)\right)
$$

which is the inequality (3). [See also [2], p159, Theorem $12 \rrbracket$

\section{References}

[1] E.K.Godunova, and V.I.Levin, On an inequality of Maroni (Russian), Mat. Zametki 2, $221-224$ (1967).

[2] D.S.Mitrinovic, Analytic Inequalities, Springer-Verlag, New York, 1970.

[3] C. Olech, "A simple proof of a certain result of Z. Opial," Ann. Polon. Math., 8, 61-63 (1960).

[4] Z. Opial, Sur une inegalite, Ann. Polon. Math., 8, 29-32 (1960).

[5] B.G.Pachpatte, "On Opial-Godunova and Levin type inequalities," Tamkang J. Math., (to appear).

[6] G.S.Yang, "On a certain result of Z. Opial," Proc. Japan Acad. 42, 78-83 (1966). 